\title{
Second Order Approximation of Possible Local Displacement for Curve Objects
}

\author{
Jun Takamatsu \\ Department of Computer Science \\ University of Tokyo \\ 7-3-1 Hongo, Bunkyo-ku \\ Tokyo 113-0033 JAPAN \\ j-taka@cvl.iis.u-tokyo.ac.jp \\ Hiroshi Kimura \\ Grad. School of Information Systems \\ Univ. of Electro-Communications \\ 1-5-1 Chofugaoka, Chofu City \\ Tokyo 182-8585 JAPAN \\ hiroshi@kimura.is.uec.ac.jp
}

\author{
Koichi Ogawara \\ Institute of Industrial Science \\ University of Tokyo \\ 4-6-1 Komaba, Meguro-ku \\ Tokyo 153-8505 JAPAN \\ ogawara@cvl.iis.u-tokyo.ac.jp \\ Katsushi Ikeuchi \\ Institute of Industrial Science \\ University of Tokyo \\ 4-6-1 Komaba, Meguro-ku \\ Tokyo 153-8505 JAPAN \\ ki@cvl.iis.u-tokyo.ac.jp
}

\begin{abstract}
Most methods for assembly tasks calculate possible local displacement of objects by the screw theory or its equivalence. The calculation is the first-order displacement approximation. Curvature information is not considered, so they can be applied to only polyhedral objects. In this paper, we propose a method to formulate and calculate the second-order approximation of possible local displacement. From our experiment, our method correctly calculated the displacement of curve objects, while most conventional methods failed.
\end{abstract}

\section{Introduction}

For recognizing assembly tasks and making automatically robot programming to execute such tasks, various methods have been proposed[1][2][3][4]. These methods require to calculate possible local displacement of objects for recognition.

To calculate this displacement, the screw theory [5] or tools with equivalent capabilities are usually employed. They are equivalent to the first order Taylor expansion of the displacement (referred to as first order displacement), and the displacement is formulated as simultaneous linear inequalities. That is a good characteristic because a powerful tool to calculate such inequalities, the theory of the polyhedral convex cones [6], has already been established.

However, the first order displacement cannot treat curvature information of objects. For example, every first order displacement of a cubic object as shown in Figure 1 is the same. That is much different from truth.

In this paper, we propose a method to formulate and calculate possible local displacement of curve objects using im-

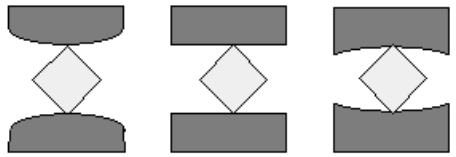

Figure 1. Same possible local displacement?

proved screw theory[7], which is equivalent to the second order Taylor expansion of the displacement (referred to as second order displacement). Then we verify the validity of a proposed method.

This paper is organized as follows: Section 2 describes preliminaries: the screw representation and the representation of curve lines and surfaces. Section 3 formulates the second order possible displacement of curve objects. Section 4 roughly introduces a method to calculate the second order displacement. Section 5 applies the proposed method to various contact relations between two curve objects. Section 6 concludes this paper.

\section{Preliminaries}

\subsection{Screw Representation}

To represent local displacement, we employ the screw representation[5]. It represents the displacement as a combination of translation along a screw axis and rotation about the same axis.

The displacement can be uniquely decided by a direction $\mathbf{r}$ and a location $\mathbf{c}$ of the screw axis, the ratio $s$ of the translation to the rotation, and a rotation angle $\Delta \theta(>0)$ in the screw representation. After the displacement, the location of any 


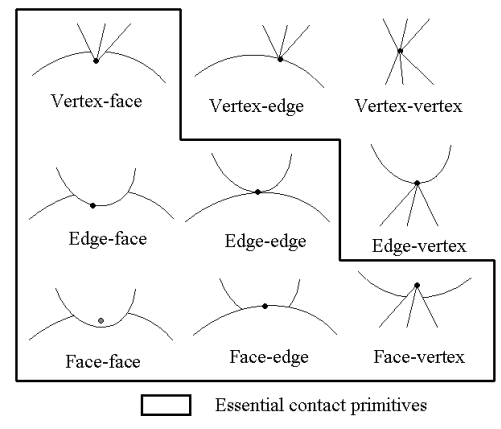

Figure 2. Nine types of contact primitives

point $\mathbf{v}$ is calculated by Equation (1).

$$
\begin{gathered}
\mathbf{v}_{m}=R(\mathbf{v}-\mathbf{c})+\mathbf{c}+s \mathbf{r} \\
R=I+\sin \Delta \theta[\mathbf{r}]_{\times}+(1-\cos \Delta \theta)[\mathbf{r}]_{\times}^{2} \\
{[\mathbf{r}]_{\times}=\left(\begin{array}{ccc}
0 & -r_{z} & r_{y} \\
r_{z} & 0 & -r_{x} \\
-r_{y} & r_{x} & 0
\end{array}\right)}
\end{gathered}
$$

In this paper, we assume that $\mathbf{c}$, and $s$ are functions with a variable $\Delta \theta^{1}$.

\subsection{Representation of Curve Line and Surface}

We assume that a curve line is parametrized by the length $s$ from a fixed point on it as Equation (2).

$$
\mathbf{x}=\mathbf{f}(s)
$$

Applying the Taylor expansion to the equation near the point, $\mathbf{p}=\mathbf{f}\left(s_{p}\right)$, Equation (3) is obtained, where $\mathbf{R}_{3}(s)$ is the third order reminder term.

$$
\mathbf{x}=\mathbf{p}+\mathbf{e}_{1} \cdot\left(s-s_{p}\right)+\frac{1}{2} \kappa \mathbf{e}_{2} \cdot\left(s-s_{p}\right)^{2}+\mathbf{R}_{3}(s)
$$

$\mathbf{e}_{1}$ is a tangent normal, $\mathbf{e}_{2}$ is a normal vector satisfied $\mathbf{e}_{1} \cdot \mathbf{e}_{2}=$ 0 , and $\kappa$ is a curvature on the point $\mathbf{p}$.

Given the function $f(\mathbf{x})$ which calculates the length between a point $\mathbf{x}$ and a surface, such a surface is formulated by $f(\mathbf{x})=0$. Applying the Taylor expansion to the equation, Equation (4) is obtained.

$$
f(\mathbf{x})=\mathbf{n} \cdot(\mathbf{x}-\mathbf{p})+\frac{1}{2}(\mathbf{x}-\mathbf{p})^{T} V(\mathbf{x}-\mathbf{p})+R_{3}(\mathbf{x})
$$

$\mathbf{n}$ is a surface normal and $V$ is a curvature matrix.

In this paper, we assume that every curve line or surface is represented as the second order approximation, that is , the equation which is obtained by removing third order reminder term $R_{3}$ from Equation (3) or (4).

\footnotetext{
${ }^{1} \mathrm{We}$ assume that $\mathbf{r}$ is fix, because rotation with a fixed axis direction is usually preferred.
}

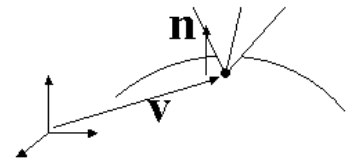

Figure 3. Vertex-face contact

\section{Second Order Displacement for Curve Lines and Surfaces}

In this paper, we consider a contact relation between two objects: one is movable and the other is fixed. An arbitrary object is composed of object primitives: vertices, edges (curve lines), and faces (curve surfaces). Every contact relation between two objects can be represented as a combination of contact primitives. A contact primitive is composed of two contacting object primitives. Therefore, nine types of contact primitives exist as shown in Figure 2. A vertex-face contact means that a vertex in a movable object contacts a face in a fixed object.

\subsection{Essential Contact Primitive}

Hirukawa et. al. proposed the method to derive the first order possible displacement from any contact relations between two polyhedral objects[9]. They derived this displacement using a contacting point and a separating plane. A separating plane composes of a contacting plane or the plane which two contacting edges is on.

And they illustrated that the first order possible displacement is represented as one system of simultaneous linear inequalities, if and only if an unique separating plane exist on every contacting point, that is, a contact relation dose not include contact primitives (referred to as singular contact primitives) as follows: 1) A convex vertex contacts a convex edge. 2) A convex vertex contacts a convex vertex.

We define following six contact primitives as essential contact primitives: vertex-face, edge-face, face-face, edge-edge, edge-face, face-face contacts (Show the area surrounded by bold line in Figure 2). That reason is as follows: 1) These six contact primitives can compose a contacting point and a unique separating curve surface. 2) Another three contact primitives can be regarded as a combination of some essential contact primitives, if they are not singular contact primitives.

In this paper, we only treat contact relations not including singular contact primitives. Therefore, the second order possible displacement can be represented by one system of simultaneous inequalities.

\subsection{Vertex-Face Contact}

Consider the case that a vertex contacts a face in the point $\mathbf{v}$ as shown in Figure 3. After the displacement, the location of the point $\mathbf{v}$ is represented by Equation (5).

$$
\mathbf{v}_{m}=R(\mathbf{v}-\mathbf{c})+\mathbf{c}+s \mathbf{r}
$$




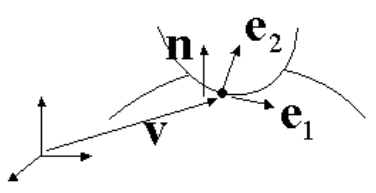

Figure 4. Edge-face contact

When a curve surface is represented as Equation (4), the length $\Delta_{v f}$ between a vertex and a curve surface after the displacement represents Equation (6).

$$
\Delta_{v f}=\mathbf{n} \cdot\left(\mathbf{v}_{m}-\mathbf{v}\right)+\frac{1}{2}\left(\mathbf{v}_{m}-\mathbf{v}\right)^{T} V\left(\mathbf{v}_{m}-\mathbf{v}\right)
$$

Applying the Taylor expansion to Equation (6), we obtain Equation (7), where $\mathbf{t}_{1}=\mathbf{r}, \mathbf{t}_{2}=\mathbf{c}(0) \times \mathbf{r}+s^{\prime}(0) \mathbf{r}, \mathbf{t}_{3}=$ $2 \mathbf{c}^{\prime}(0) \times \mathbf{r}+s^{\prime \prime}(0) \mathbf{r}$.

$$
\begin{aligned}
\Delta_{v f}= & \mathbf{n} \cdot\left(\mathbf{t}_{1} \times \mathbf{v}+\mathbf{t}_{2}\right) \Delta \theta \\
+ & \left(\mathbf{n} \cdot \mathbf{t}_{3}+\left(\mathbf{n} \times \mathbf{t}_{1}\right) \cdot\left(\mathbf{t}_{1} \times \mathbf{v}+\mathbf{t}_{2}\right)\right. \\
& \left.+\left(\mathbf{t}_{1} \times \mathbf{v}+\mathbf{t}_{2}\right)^{T} V\left(\mathbf{t}_{1} \times \mathbf{v}+\mathbf{t}_{2}\right)\right) \frac{\Delta \theta^{2}}{2} \\
+ & R_{3}(\Delta \theta)
\end{aligned}
$$

The possible displacement is formulated by $\Delta_{v f} \geq 0$.

\subsection{Edge-Face Contact}

Consider the case that an edge contacts a face in the point $\mathbf{v}$ as shown in Figure 3. We assume that a curve line is represented by Equation (8).

$$
\mathbf{e}(s)=\mathbf{v}+s \mathbf{e}_{1}+\frac{1}{2} s^{2} \kappa \mathbf{e}_{2}
$$

After the displacement, a curve line is represented by Equation (9).

$$
\mathbf{e}_{m}(s)=\mathbf{v}_{m}+s R \mathbf{e}_{1}+\frac{1}{2} s^{2} \kappa R \mathbf{e}_{2}
$$

When a curve surface is represented as Equation (4), the length $\Delta_{e f}$ between a point on the edge, $\mathbf{e}_{m}\left(s_{p}\right)$, and the curve surface is represented by Equation (10).

$$
\begin{aligned}
\Delta_{e f} & =\mathbf{n} \cdot\left(\mathbf{e}_{m}\left(s_{p}\right)-\mathbf{v}\right) \\
& +\frac{1}{2}\left(\mathbf{e}_{m}\left(s_{p}\right)-\mathbf{v}\right)^{T} V\left(\mathbf{e}_{m}\left(s_{p}\right)-\mathbf{v}\right)
\end{aligned}
$$

When the length is minimum, Equation (11) must be satisfied. Such minimum length is the length between an edge and a face.

$$
\frac{\partial \Delta_{e f}}{\partial s_{p}}=0
$$

And, Equation (12) must be satisfied, because an edge contacts a face without penetrating.

$$
\mathbf{n} \cdot \mathbf{e}_{1}=0
$$

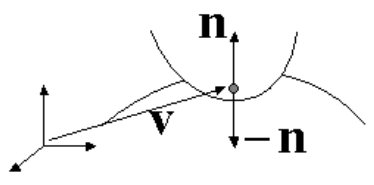

Figure 5. Face-face contact

Applying the Taylor expansion to Equation (10) and using Equation (11) and (12), we obtain Equation (13).

$$
\begin{aligned}
& \Delta_{e f}= \mathbf{n} \cdot\left(\mathbf{t}_{1} \times \mathbf{v}+\mathbf{t}_{2}\right) \Delta \theta \\
&+\left(\mathbf{n} \cdot \mathbf{t}_{3}+\left(\mathbf{n} \times \mathbf{t}_{1}\right) \cdot\left(\mathbf{t}_{1} \times \mathbf{v}+\mathbf{t}_{2}\right)\right. \\
&+\left(\mathbf{t}_{1} \times \mathbf{v}+\mathbf{t}_{2}\right)^{T} V\left(\mathbf{t}_{1} \times \mathbf{v}+\mathbf{t}_{2}\right) \\
&-a^{2}\left(\kappa \mathbf{n} \cdot \mathbf{e}_{2}+\mathbf{e}_{1}^{T} V \mathbf{e}_{1}\right) \frac{\Delta \theta^{2}}{2} \\
&+ R_{3}(\Delta \theta) \\
& a=-\left(\mathbf{e}_{1} \times \mathbf{n}\right) \cdot \mathbf{t}_{1}+\mathbf{e}_{1}^{T} V\left(\mathbf{t}_{1} \times \mathbf{v}+\mathbf{t}_{2}\right) \\
& \kappa \mathbf{n} \cdot \mathbf{e}_{2}+\mathbf{e}_{1}^{T} V \mathbf{e}_{1}
\end{aligned}
$$

The possible displacement is formulated by $\Delta_{e f} \geq 0$.

\subsection{Face-Face Contact}

Consider the case that one face contacts the other face in the point $\mathbf{v}$ as shown in Figure 5. We assume that two faces are represented by Equation (14).

$$
\begin{array}{r}
f(\mathbf{x})=-\mathbf{n} \cdot(\mathbf{x}-\mathbf{v})+\frac{1}{2}(\mathbf{x}-\mathbf{v})^{T} V_{f}(\mathbf{x}-\mathbf{v})=0 \\
g(\mathbf{x})=\mathbf{n} \cdot(\mathbf{x}-\mathbf{v})+\frac{1}{2}(\mathbf{x}-\mathbf{v})^{T} V_{g}(\mathbf{x}-\mathbf{v})=0
\end{array}
$$

After the displacement, the curve surface, $f(\mathbf{x})=0$, is represented by Equation (15).

$$
\begin{aligned}
f_{m}(\mathbf{x}) & =-R \mathbf{n} \cdot(\mathbf{x}-\mathbf{v}) \\
& +\frac{1}{2}(\mathbf{x}-\mathbf{v})^{T} R V_{f} R^{T}(\mathbf{x}-\mathbf{v})=0
\end{aligned}
$$

Let $\mathbf{p}$ be any point on the surface $g(\mathbf{x})=0$. We assume that $\mathbf{p}$ is represented as Equation (16), where $\mathbf{k}_{1}$ and $\mathbf{k}_{2}$ are two tangent vector of the surface with maximum and minimum curvature, and $\mathbf{k}_{1} \times \mathbf{k}_{2}=\mathbf{n}, \mathbf{k}_{2} \times \mathbf{n}=\mathbf{k}_{1}, \mathbf{n} \times \mathbf{k}_{1}=\mathbf{k}_{2}$ are satisfied.

$$
\mathbf{s}=q_{1} \mathbf{k}_{1}+q_{2} \mathbf{k}_{2}+q_{3} \mathbf{n}+\mathbf{v}
$$

Because $\mathbf{p}$ is on the surface, Equation (17) must be satisfied, where $\kappa_{1}$ and $\kappa_{2}$ are maximum and minimum curvature.

$$
q_{3}=-\frac{1}{2} \kappa_{1} q_{1}^{2}-\frac{1}{2} \kappa_{2} q_{2}^{2}
$$

The length between two face is equal to the minimum of Equation (18).

$$
\Delta_{f f}=f_{m}(\mathbf{p})
$$




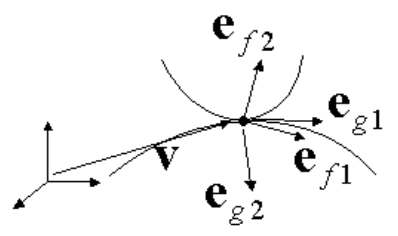

Figure 6. Edge-edge contact

If $\Delta_{f f}$ is minimum, Equation (19) must be satisfied.

$$
\frac{\partial \Delta_{f f}}{\partial q_{1}}=0, \frac{\partial \Delta_{f f}}{\partial q_{2}}=0
$$

Applying the Taylor expansion to Equation (18) and using Equation (17) and (19), Equation (20) is obtained, where $V_{\text {diff }}=V_{f}-V_{g}$ and $K=\left(\mathbf{k}_{1} \mathbf{k}_{2}\right)$

$$
\begin{aligned}
& \Delta_{f f}= \mathbf{n} \cdot\left(\mathbf{t}_{1} \times \mathbf{v}+\mathbf{t}_{\mathbf{2}}\right) \Delta \theta \\
&+\left(\mathbf{n} \cdot \mathbf{t}_{3}-\left(\mathbf{n} \times \mathbf{t}_{1}\right) \cdot\left(\mathbf{t}_{1} \times \mathbf{v}+\mathbf{t}_{2}\right)\right. \\
&+\left(\mathbf{t}_{1} \times \mathbf{v}+\mathbf{t}_{2}\right)^{T} V_{f}\left(\mathbf{t}_{1} \times \mathbf{v}+\mathbf{t}_{2}\right) \\
&-\mathbf{n} \cdot \mathbf{b}) \frac{\Delta \theta^{2}}{2}+R_{3}(\Delta \theta) \\
& \mathbf{b}= \kappa_{1} c_{1}^{2}+\kappa_{2} c_{2}^{2} \\
&\left(\begin{array}{c}
c_{1} \\
c_{2}
\end{array}\right)=M^{-} \mathbf{d} \\
& M= K^{T} V_{\text {diff }} K \\
& \mathbf{d}=\left(\begin{array}{l}
\mathbf{k}_{1}^{T} V_{f}\left(\mathbf{t}_{\mathbf{1}} \times \mathbf{v}+\mathbf{t}_{\mathbf{2}}\right)-\mathbf{k}_{2} \cdot \mathbf{t}_{1} \\
\mathbf{k}_{2}^{T} V_{f}\left(\mathbf{t}_{\mathbf{1}} \times \mathbf{v}+\mathbf{t}_{\mathbf{2}}\right)+\mathbf{k}_{1} \cdot \mathbf{t}_{1}
\end{array}\right)
\end{aligned}
$$

The possible displacement is formulated by $\Delta_{f f} \geq 0$.

\subsection{Edge-Edge Contact}

In this paper, we define the length between two edges as Equation (21), where $\mathbf{v}_{1}$ and $\mathbf{v}_{2}$ are points on edges of moving and fixed objects, $\mathbf{t}_{1}$ and $\mathbf{t}_{2}$ are tangent vectors on $\mathbf{v}_{1}$ and $\mathbf{v}_{2}$, and a direction of the vector $\mathbf{t}_{1} \times \mathbf{t}_{2}(\neq \mathbf{0})$ is outward to a fixed object.

$$
\min _{\mathbf{v}_{1}, \mathbf{v}_{2}}\left(\mathbf{t}_{1} \times \mathbf{t}_{2}\right) \cdot\left(\mathbf{v}_{1}-\mathbf{v}_{2}\right)
$$

When the equation is equal to 0 , two edges contact each other. And when the equation is less than 0 , they penetrate each other.

Consider the case that one edge contacts the other edge in the point $\mathbf{v}$ as shown in Figure 6. We assume that two curve lines are represented by Equation (22).

$$
\begin{aligned}
& \mathbf{f}(l)=\mathbf{v}+l \mathbf{e}_{f 1}+\frac{1}{2} l^{2} \kappa \mathbf{e}_{f 2} \\
& \mathbf{g}(l)=\mathbf{v}+l \mathbf{e}_{g 1}+\frac{1}{2} l^{2} \kappa \mathbf{e}_{g 2}
\end{aligned}
$$

After the displacement, the curve line $\mathbf{f}(l)$ is represented by Equation (23).

$$
\mathbf{f}_{m}(l)=\mathbf{v}_{m}+l R \mathbf{e}_{f 1}+\frac{1}{2} l^{2} \kappa R \mathbf{e}_{f 2}
$$

First, we consider the case that two edges are not locally on the same plane. In this case, Equation (24) is satisfied.

$$
\mathbf{e}_{f 1} \times \mathbf{e}_{g 1} \neq 0
$$

The length between two edges is equal to the minimum of Equation (25).

$$
\Delta_{e e}=\left(\frac{\partial \mathbf{f}_{m}\left(l_{1}\right)}{\partial l_{1}} \times \frac{\partial \mathbf{g}\left(l_{2}\right)}{\partial l_{2}}\right) \cdot\left(\mathbf{f}_{m}\left(l_{1}\right)-\mathbf{g}\left(l_{2}\right)\right)
$$

If $\Delta_{e e}$ is minimum, Equation (26) must be satisfied.

$$
\frac{\partial \Delta_{e e}}{\partial l_{1}}=0, \frac{\partial \Delta_{e e}}{\partial l_{2}}=0
$$

Applying the Taylor expansion to Equation (25) and using Equation (26), we obtain Equation (27).

$$
\begin{aligned}
\Delta_{e e} & =\mathbf{n} \cdot \mathbf{t}_{3}+\left(\mathbf{n} \times \mathbf{t}_{1}\right) \times\left(\mathbf{t}_{1} \times \mathbf{v}+\mathbf{t}_{2}\right) \\
& +2\left(\left(\mathbf{t}_{1} \times \mathbf{e}_{f 1}\right) \times \mathbf{e}_{g 1}\right) \cdot\left(\mathbf{t}_{1} \times \mathbf{v}+\mathbf{t}_{2}\right) \\
& +\mathbf{n} \cdot\left(\kappa_{f} a_{1}^{2} \mathbf{e}_{f 2}-\kappa_{g} a_{2}^{2} \mathbf{e}_{g 2}\right) \\
a_{1} & =\frac{\left(\mathbf{e}_{f 2} \times \mathbf{e}_{g 1}\right) \cdot\left(\mathbf{t}_{1} \times \mathbf{v}+\mathbf{t}_{2}\right)}{\left(\mathbf{e}_{f 1} \times \mathbf{e}_{g 1}\right) \cdot \mathbf{e}_{f 2}} \\
a_{2} & =\frac{\left(\mathbf{e}_{g 2} \times \mathbf{e}_{f 1}\right) \cdot\left(\mathbf{t}_{1} \times \mathbf{v}+\mathbf{t}_{2}\right)}{\left(\mathbf{e}_{f 1} \times \mathbf{e}_{g 1}\right) \cdot \mathbf{e}_{g 2}}
\end{aligned}
$$

The possible displacement is formulated by $\Delta_{e e} \geq 0$.

When Equation (24) is not satisfied, this contact primitive can be regarded as a combination of another contact primitives as the case of polyhedral objects.

\subsection{Face-Vertex and Face-Edge Contact}

Consider the case that a face contacts a vertex in one point. Viewing the displacement from the vertex, the face inversely displaces. From the definition, the inverse displacement can be represented as $-\mathbf{t}_{1},-\mathbf{t}_{2},-\mathbf{t}_{3}$. Therefore, the equation to represent the second order displacement is obtained by substituting $-\mathbf{t}_{1},-\mathbf{t}_{2},-\mathbf{t}_{3}$ to Equation (7). As the same way, we obtain the equation to represent the second order displacement in a face-edge contact case is obtained.

\section{Calculating the Second Order Displacement}

The second order possible displacement can be calculated by investigating signs of coefficients of $\Delta \theta$ and $\Delta \theta^{2}$ as shown in Table 1.

First, we calculate the first order displacement. Then, we calculate the second order displacement by classifying the first order reciprocal motion. For calculating the first order displacement, various methods have been proposed[10][11]. We proposed the method to classify the first order reciprocal motion into the second order reciprocal motion or not[7]. However, it is so difficult to classify the first order reciprocal motion into the second order repelling, reciprocal, or contrary 
Table 1. Second order displacement

\begin{tabular}{|c|c|c|}
\hline $\begin{array}{l}\text { coefficient } \\
\text { of } \Delta \theta\end{array}$ & $\begin{array}{l}\text { coefficient } \\
\text { of } \Delta \theta^{2}\end{array}$ & $\begin{array}{c}\text { possible } \\
\text { displacement }\end{array}$ \\
\hline $\begin{array}{c}>0 \\
\text { (first order } \\
\text { repelling) }\end{array}$ & - & \multirow{3}{*}{ possible } \\
\hline \multirow{3}{*}{$\begin{array}{l}\quad=0 \\
\text { (first order } \\
\text { reciprocal) }\end{array}$} & $\begin{array}{c}>0 \\
\text { (second order } \\
\text { repelling) }\end{array}$ & \\
\hline & $\begin{array}{c}=0 \\
\text { (second order } \\
\text { reciprocal) }\end{array}$ & \\
\hline & $\begin{array}{c}<0 \\
\text { (second order } \\
\text { contrary) }\end{array}$ & \multirow[b]{2}{*}{ impossible } \\
\hline $\begin{array}{c}<0 \\
\text { (first order } \\
\text { contrary) }\end{array}$ & - & \\
\hline
\end{tabular}

motions. That is an open problem. Therefore, we roughly introduce a method to classify the first order reciprocal motion into the second order reciprocal motion or not.

Because the first order possible displacement is represented as one system of simultaneous linear inequalities, the first order reciprocal motion is represented as a linear sum of some bases as shown in Equation (28), where $a_{i}$ is any real number.

$$
\left[\mathbf{t}_{1}, \mathbf{t}_{2}\right]=\sum a_{i} \mathbf{b}_{\mathbf{i}}
$$

Substituting the equation to coefficients of $\Delta \theta^{2}$, they are generally represented as Equation (29), where $h_{i}\left(a_{1}, \ldots, a_{r}\right)$ is the second order homogeneous equation.

$$
\mathbf{n}_{i} \cdot \mathbf{t}_{3}+h_{i}\left(a_{1}, \ldots, a_{r}\right)
$$

To classify the first order reciprocal motion into the second order reciprocal motion or not, we need to solve Equation (30).

$$
\bigcap \mathbf{n}_{i} \cdot \mathbf{t}_{3}+h_{i}\left(a_{1}, \ldots, a_{r}\right)=0
$$

Searching linear dependent combinations of $\left\{\mathbf{n}_{i}\right\}$, equations including $h_{i}$ only are obtained. Such equations are naturally the second order homogeneous. Next we decompose these equations to linear equations: 1) If it is equivalent to $A_{1}^{2}+\cdots+A_{n}^{2}=0$, it can be decomposed into Equation (31).

$$
\bigcap_{i}^{n} A_{i}=0
$$

2) If it is equivalent to $A_{1}^{2}-A_{2}^{2}=0$, it can be decomposed into Equation (32).

$$
A_{1}+A_{2}=0 \bigcup A_{1}-A_{2}=0
$$

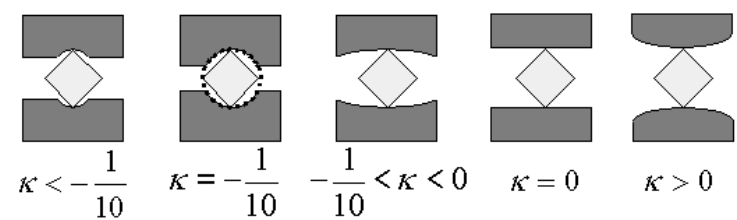

Figure 7. Example

\section{Example}

We apply the proposed method to a contact relation consisting of four vertex-face contacts as shown in Figure 7. We assume that locations of four vertices are $(0,20,10)$, $(0,20,-10),(0,-20,10)$, and $(0,-20,-10)$, surface normals of two faces are $(0,0,1)$ and $(0,0,-1)$, curvatures of two faces along the $\mathrm{y}$-axis are 0 , and curvatures along the $\mathrm{x}$ axis are $\kappa$.

First, we formulate the first order possible displacement as Equation (33).

$$
\left(\begin{array}{cccccc}
10 & 0 & 0 & 0 & 0 & -1 \\
-10 & 0 & 0 & 0 & 0 & 1 \\
-10 & 0 & 0 & 0 & 0 & -1 \\
10 & 0 & 0 & 0 & 0 & 1
\end{array}\right)\left(\begin{array}{l}
\mathbf{t}_{\mathbf{1}} \\
\mathbf{t}_{\mathbf{2}}
\end{array}\right) \geq \mathbf{0}
$$

We obtain Equation (34) by solving Equation (33). The solution means an object cannot translate along the z-axis and rotate about the $\mathrm{x}$-axis, and other displacements are the first order reciprocal motions.

$$
\left(\begin{array}{cccccc}
10 & 0 & 0 & 0 & 0 & -1 \\
-10 & 0 & 0 & 0 & 0 & -1
\end{array}\right)\left(\begin{array}{l}
\mathbf{t}_{\mathbf{1}} \\
\mathbf{t}_{\mathbf{2}}
\end{array}\right)=\mathbf{0}
$$

We classify all the first order reciprocal motions into the second order reciprocal motions or not. We select bases of the first order reciprocal motions as Equation (35)

$$
\begin{array}{r}
\mathbf{s}_{\mathbf{1}}=(0,1,0,0,0,0), \mathbf{s}_{\mathbf{2}}=(0,0,0,1,0,0) \\
\mathbf{s}_{\mathbf{3}}=(0,0,0,0,1,0), \mathbf{s}_{\mathbf{4}}=(0,0,-1,0,0,0)
\end{array}
$$

We obtain Equation (36) which the second order reciprocal motion must be satisfied (See the next page).

We obtain Equation (37) to (40) by searching linear dependent combinations.

$$
\begin{aligned}
20(1+10 \kappa) s_{1}^{2}+2 \kappa s_{2}^{2}+800 \kappa s_{4}^{2} & =0 \\
40(1+20 \kappa) s_{1} s_{4}+80 \kappa s_{2} s_{4} & =0 \\
20(1+10 \kappa) s_{1}^{2}+40(1+20 \kappa) s_{1} s_{4} & \\
+2 \kappa s_{2}^{2}+80 \kappa s_{2} s_{4}+800 \kappa s_{4}^{2} & =0 \\
20(1+10 \kappa) s_{1}^{2}-40(1+20 \kappa) s_{1} s_{4} & \\
+2 \kappa s_{2}^{2}-80 \kappa s_{2} s_{4}+800 \kappa s_{4}^{2} & =0
\end{aligned}
$$

When $\kappa>0, s_{1}=s_{2}=s_{4}=0$ is obtained from Equation (37). That means only translation along the y-axis can maintain the contact relation. 


$$
\begin{array}{r}
(0,0,-1) \cdot \mathbf{t}_{\mathbf{3}}+10(1+10 \kappa) s_{1}^{2}+(1+20 \kappa) s_{1} s_{2}+20(1+20 \kappa) s_{1} s_{4}+\kappa s_{2}^{2}+40 \kappa s_{2} s_{4}+400 \kappa s_{4}^{2}=0 \\
(0,0,1) \cdot \mathbf{t}_{\mathbf{3}}+10(1+10 \kappa) s_{1}^{2}-(1+20 \kappa) s_{1} s_{2}+20(1+20 \kappa) s_{1} s_{4}+\kappa s_{2}^{2}+40 \kappa s_{2} s_{4}+400 \kappa s_{4}^{2}=0 \\
(0,0,-1) \cdot \mathbf{t}_{\mathbf{3}}+10(1+10 \kappa) s_{1}^{2}+(1+20 \kappa) s_{1} s_{2}-20(1+20 \kappa) s_{1} s_{4}+\kappa s_{2}^{2}-40 \kappa s_{2} s_{4}+400 \kappa s_{4}^{2}=0 \\
(0,0,1) \cdot \mathbf{t}_{\mathbf{3}}+10(1+10 \kappa) s_{1}^{2}-(1+20 \kappa) s_{1} s_{2}-20(1+20 \kappa) s_{1} s_{4}+\kappa s_{2}^{2}-40 \kappa s_{2} s_{4}+400 \kappa s_{4}^{2}=0
\end{array}
$$

When $\kappa=0, s_{1}=0$ is obtained from Equation (37). That means rotation about the $y$-axis cannot maintain the contact relation.

When $-1 / 10<\kappa<0, s_{4}=0 \bigcup(1+20 \kappa) s_{1}+$ $2 \kappa s_{2}=0$ is obtained from Equation (38). 1) When $s_{4}=0$, $\sqrt{10(1+10 \kappa)} s_{1}+\sqrt{\kappa} s_{2}=0 \bigcup \sqrt{10(1+10 \kappa)} s_{1}-\sqrt{\kappa} s_{2}=$ 0 is obtained form Equation (37). That means translation along $\mathrm{x}$ axis is necessary for rotation about the $\mathrm{y}$-axis to maintain the contact relation. 2) When $(1+20 \kappa) s_{1}+2 \kappa s_{2}=0$, it is difficult to analyze what the result means. The result represents the case that rotation axis is on the xz-plane. In this case, one cannot solve the answer intuitively.

When $\kappa=-1 / 10, s_{2}=s_{4}=0$ is obtained from Equation (37). That means translation along the $\mathrm{x}$-axis and rotation about the $\mathrm{z}$-axis cannot maintain the contact relation.

When $\kappa<-1 / 10, s_{1}=s_{2}=s_{4}=0$ is obtained from Equation (37), the result is the same as $\kappa 0>0$.

\section{Conclusion}

In this paper, we proposed a method to formulate and calculate the second order displacement. First, we formulate the second order displacement in each contact primitives. These formulas are very different from each other, however, they can be treated as the same way when calculating.

Then, we verified the proposed method by using the case that four vertex-face contacts exist. We illustrated that a solution of calculating the second order displacement is changed in concord with the change of the face curvature. We found that the solution is correct and the proposed method is superior to the original screw theory.

\section{ACKNOWLEDGMENTS}

This work is supported in part by the Japan Science and Technology Corporation (JST) under the Ikeuchi CREST project, and in part by the Grant-in-Aid for Scientific Research on Priority Areas (C) 14019027 of the Ministry of Education, Culture, Sports, Science and Technology.

\section{References}

[1] K. Ikeuchi and T. Suehiro : "Toward an assembly plan from observation part i: Task recognition with polyhedral objects," IEEE Trans. on Robotics and Automation, Vol. 10, No. 3, Jun. 1994.
[2] Y. Kuniyoshi, M. Inaba, and H. Inoue : "Learning by watching: Extracting reusable task knowledge from visual observation of human performance," IEEE Trans. on Robotics and Automation, Vol. 10, No. 6, Dec. 1994.

[3] M. Tsuda, T. Takahashi, and H. Ogata : "Generation of an assembly-task model analyzing human demonstration," Journal of the Robotics Society of Japan, Vol. 18, No. 4, pp. $535-544,2000$.

[4] H. Onda, T. Ogasawara, H. Hirukawa, K. Kitagaki, A. Nakamura, and H. Tsukune : "A telerobotics system using planning functions based on manipulation skills and teaching-by-demonstration technique in vr," Journal of the Robotics Society of Japan, Vol. 18, No. 7, pp. 979 - 994, 2000.

[5] M. S. Ohwovoriole and B. Roth : "An extension of screw theory," Journal of Mechanical Design, Vol. 103, pp. 725 - 735, Oct. 1981.

[6] H. W. Kuhn and A. W. Tucker : "Linear inequalities and related systems," Annals. of Mathematics Studies, Vol. 38, , 1956.

[7] J. Takamatsu, H. Kimura, and K. Ikeuchi : "Improved screw theory using second order terms," IEEE Inter. Conf. on Intelligent Robots and Systems, pp. 1614 1618, 2002.

[8] E. Rimon and J. Burdick : "Mobility of bodies in contact - i: A new 2nd order mobility index for multiple-finger grasps," IEEE Inter. Conf. on Robotics and Automation, pp. $2329-2355,1994$.

[9] H. Hirukawa, T. Matsui, and K. Takase : "A general algorithm for deriving constraint of contact between polyhedra from geometric model," Journal of the Robotics Society of Japan, Vol. 9, No. 4, pp. 415 - 426, 1991.

[10] H. Hirukawa, T. Matsui, and K. Takase : "A fast algorithm for the analysis of the constraint for motion of polyhedra in contact and its application to departure motion planning," Journal of the Robotics Society of Japan, Vol. 9, No. 7, pp. 841 - 848, 1991.

[11] S. Hirai : "Kinematics and statics of manipulation using the theory of polyhedral convex cones and their application to the planning of manipulative operations," Journal of the Robotics Society of Japan, Vol. 17, No. 1, pp. $68-83,1999$. 